Mahdi Asgari · Ralf Schmidt

\title{
Siegel modular forms and representations
}

Received: 28 March 2000 / Revised version: 25 October 2000

\begin{abstract}
This paper explicitly describes the procedure of associating an automorphic representation of $\operatorname{PGSp}(2 n, \mathbb{A})$ with a Siegel modular form of degree $n$ for the full modular group $\Gamma_{n}=\operatorname{Sp}(2 n, \mathbb{Z})$, generalizing the well-known procedure for $n=1$. This will show that the so-called "standard" and "spinor" $L$-functions associated with such forms are obtained as Langlands $L$-functions. The theory of Euler products, developed by Langlands, applied to a Levi subgroup of the exceptional group of type $F_{4}$, is then used to establish meromorphic continuation for the spinor $L$-function when $n=3$.
\end{abstract}

\section{Introduction}

Let $f$ be a Siegel modular form of degree $n$ for the full modular group $\Gamma_{n}=$ $\operatorname{Sp}(2 n, \mathbb{Z})$. If $f$ is an eigenfunction for the action of the Hecke algebra, then there are two $L$-functions associated with $f$. Let $a_{0}, a_{1}, \ldots, a_{n}$ be the Satake parameters of $f$, and define the standard L-function

$$
L_{1}(s, f)=\prod_{p}\left(\left(1-p^{-s}\right) \prod_{i=1}^{n}\left(1-a_{i} p^{-s}\right)\left(1-a_{i}^{-1} p^{-s}\right)\right)^{-1},
$$

and the spinor L-function

$$
L_{2}(s, f)=\prod_{p}\left(\prod_{k=0}^{n} \prod_{1 \leq i_{1}<\ldots<i_{k} \leq n}\left(1-a_{0} a_{i_{1}} \ldots a_{i_{k}} p^{-s}\right)\right)^{-1} .
$$

One goal of this note is to "explain" the definition of these $L$-functions within the general framework of automorphic representations. To do so, we first associate an automorphic representation with the classical modular form $f$. We then identify the above $L$-functions with certain Langlands $L$-functions coming from two different representations of the dual group. Langlands' theory of Euler products will then imply the following (cf. Sect. 4.6).

M. Asgari: Department of Mathematics, The University of Michigan, Ann Arbor, MI 481091109, USA.

e-mail: asgari@math.lsa.umich.edu

R. Schmidt: Universität des Saarlandes, Fachrichtung 6.1 Mathematik, Postfach 1511 50, 66041 Saarbrücken, Germany.

e-mail: rschmidt@math.uni-sb.de

Mathematics Subject Classification (2000): 11F46 
Theorem. The L-functions $L_{1}(s, f)$ and $L_{2}(s, f)$ have meromorphic continuation to all of $\mathbb{C}$ when $n=3$.

We should remark that Böcherer has proved stronger results for the standard $L$-functions (cf. [Bö]). Andrianov also gives all the analytic properties of spinor $L$-function when $n=2$ in [An].

The procedure of associating an automorphic representation to a classical modular form is well known in the case of elliptic modular forms, see [Ge] Chapter 3 or [Bu, Sect. 3.6]. There, one associates with an eigenform $f$ an automorphic representation $\pi_{f}$ of $\operatorname{GL}(2, \mathbb{A})$, where $\mathbb{A}$ denotes the adeles of $\mathbb{Q}$. If $f$ has no character, then $\pi_{f}$ will have trivial central character, thus descends to a representation of $\operatorname{PGL}(2, \mathbb{A})$. In the higher dimensional case we will associate with an eigenform $f$ of degree $n$ an automorphic representation of the group $\operatorname{GSp}(2 n, \mathbb{A})$ which is isomorphic to $\operatorname{GL}(2, \mathbb{A})$ if $n=1$. Since $f$ will be assumed to have no character, this is really a representation of $\operatorname{PGSp}(2 n, \mathbb{A})$.

To be more precise, utilizing a strong approximation theorem, we first lift $f$ to a function $\Phi_{f}$ on $G(\mathbb{A})$, where $G=\operatorname{GSp}(2 n)$. We will assume $f$ to be cuspidal, so that $\Phi_{f}$ lies in the cuspidal subspace $L_{0}^{2}(G(\mathbb{Q}) \backslash G(\mathbb{A})$ ). (Here we have to translate the classical cusp condition into the group theoretic one.) Then let $\pi$ be the subrepresentation of this space generated by $\Phi_{f}$. This $\pi$ may not be irreducible, but if $f$ is an eigenform, then all the irreducible components of $\pi$ will turn out to be isomorphic. This isomorphism class is the automorphic representation $\pi_{f}$ associated with $f$. In Theorem 2 we shall describe its local components in terms of Satake parameters (at the finite places) and Harish-Chandra parameters (at the infinite place).

We have to go through some Hecke algebra computations to identify the classical Satake parameters of the eigenform $f$ with the Satake parameters of the local components of $\pi_{f}$ which are spherical representations of the local groups $\operatorname{GSp}\left(2 n, \mathbb{Q}_{p}\right)$. The group theoretic Satake parameters can be taken to be in the maximal torus in the dual group of $G$ which is $\hat{G}=\operatorname{GSpin}(2 n+1, \mathbb{C})$. Since we have a representation with trivial central character, the Satake parameters will in fact be elements of its derived group $\operatorname{Spin}(2 n+1, \mathbb{C})$.

This latter group has two distinguished finite-dimensional representations, namely the projection onto $\operatorname{SO}(2 n+1, \mathbb{C})$ which we denote by $\varrho_{1}$, and the $2^{n}$ dimensional spin representation $\varrho_{2}$, the smallest genuine representation (not factoring through $\varrho_{1}$ ) of this group. We use the weight structure of these finite-dimensional representations to determine the form of the standard Euler factor associated with $\varrho_{1}$ and $\varrho_{2}$. It turns out that these Euler factors are precisely the same as the ones in (1) and (2). In other words, the classical $L$-functions identify with standard Langlands $L$-functions coming from the representations $\varrho_{1}$ and $\varrho_{2}$ of the dual group (see Theorem 3).

Having established this identification, one can apply results from representation theory to classical modular forms. For modular forms of degree 3, the underlying group GSp(6) can be embedded, as a Levi, in a Chevalley group of type $F_{4}$. We then make use of Langlands' method developed in [La] to prove the meromorphic continuation of the spin $L$-function for $n=3$ (cf. Corollary 1). 


\section{Notation}

The right group for handling Siegel modular forms (of degree $n$ ) in the context of automorphic representations is

$$
G=\mathrm{GSp}(2 n)=\left\{g \in \mathrm{GL}(2 n): \exists \mu(g) \in \mathrm{GL}(1) g J^{t} g=\mu(g) J\right\},
$$

where

$$
J=\left(\begin{array}{c}
\mathbf{1}_{n} \\
-\mathbf{1}_{n}
\end{array}\right), \quad \mathbf{1}_{n} \text { the } n \times n \text { identity matrix. }
$$

If $g=\left(\begin{array}{ll}A & B \\ C & D\end{array}\right)$, then the conditions are equivalent to

$$
A^{t} D-B{ }^{t} C=\mu(g) \mathbf{1}, \quad A^{t} B=B{ }^{t} A, \quad C^{t} D=D^{t} C .
$$

The function $\mu$ is called the multiplier homomorphism. Its kernel is the group $\operatorname{Sp}(2 n)$ and there is an exact sequence

$$
1 \longrightarrow \mathrm{Sp}(2 n) \longrightarrow G \longrightarrow \mathrm{GL}(1) \longrightarrow 1 .
$$

The center $Z$ of $G$ consists of the scalar matrices, and the standard maximal torus is

$$
T=\left\{\operatorname{diag}\left(u_{1}, \ldots, u_{n}, v_{1}, \ldots, v_{n}\right): u_{1} v_{1}=\ldots=u_{n} v_{n} \neq 0\right\} .
$$

We often write an element $t \in T$ in the form

$$
t=\operatorname{diag}\left(u_{1}, \ldots, u_{n}, u_{1}^{-1} u_{0}, \ldots, u_{n}^{-1} u_{0}\right), \quad u_{i} \in \mathrm{GL}(1) ;
$$

then $u_{0}=\mu(t)$. We fix the following characters of the maximal torus $T \subset G$. If $t \in T$ is written in the form (3), then let

$$
e_{i}(t)=u_{i}, \quad i=0,1, \ldots, n .
$$

These characters are a basis for the character lattice of $G$,

$$
X=\mathbb{Z} e_{0} \oplus \mathbb{Z} e_{1} \oplus \ldots \oplus \mathbb{Z} e_{n} .
$$

We also fix the following cocharacters of $T$ :

$$
\begin{aligned}
& f_{0}(u)=\operatorname{diag}(\underbrace{1, \ldots, 1}_{n}, \underbrace{u, \ldots, u}_{n}), \\
& f_{1}(u)=\operatorname{diag}(\underbrace{u, 1, \ldots, 1}_{n}, \underbrace{u^{-1}, 1, \ldots, 1}_{n}), \\
& \vdots \\
& f_{n}(u)=\operatorname{diag}(\underbrace{1, \ldots, 1, u}_{n}, \underbrace{1, \ldots, 1, u^{-1}}_{n}) .
\end{aligned}
$$


Then these elements are a $\mathbb{Z}$-basis for the cocharacter lattice of $G$,

$$
X^{\vee}=\mathbb{Z} f_{0} \oplus \mathbb{Z} f_{1} \oplus \ldots \oplus \mathbb{Z} f_{n} .
$$

With the natural pairing $\langle\rangle:, X \times X^{\vee} \longrightarrow \mathbb{Z}$, we have

$$
\left\langle e_{i}, f_{j}\right\rangle=\delta_{i j} .
$$

We now choose the following set of simple roots:

$$
\alpha_{1}(t)=u_{n-1}^{-1} u_{n}, \quad \ldots \quad, \alpha_{n-1}(t)=u_{1}^{-1} u_{2}, \quad a_{n}(t)=u_{1}^{2} u_{0}^{-1} ;
$$

here $t$ is written in the form (3). In other words,

$$
\alpha_{1}=e_{n}-e_{n-1}, \quad \ldots, \quad \alpha_{n-1}=e_{2}-e_{1}, \quad \alpha_{n}=2 e_{1}-e_{0} .
$$

The numbering of the simple roots is such that the Dynkin diagram is

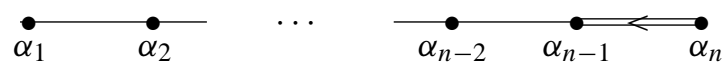

The corresponding coroots are

$$
\alpha_{1}^{\vee}=f_{n}-f_{n-1}, \quad \ldots \quad \alpha_{n-1}^{\vee}=f_{2}-f_{1}, \quad \alpha_{n}^{\vee}=f_{1} .
$$

If we let $R=\left\{\alpha_{1}, \ldots, \alpha_{n}\right\} \subset X, R^{\vee}=\left\{\alpha_{1}^{\vee}, \ldots, \alpha_{n}^{\vee}\right\} \subset X^{\vee}$, then

$$
\left(X, R, X^{\vee}, R^{\vee}\right)
$$

is the root datum of $G=\operatorname{GSp}(2 n)$. The Cartan matrix is

$$
\left\langle\alpha_{i}, \alpha_{j}^{\vee}\right\rangle=\left(\begin{array}{cccccccc}
2 & -1 & & & & & & \\
-1 & 2 & -1 & & & & \\
& -1 & 2 & -1 & & & \\
& & \ddots & \ddots & \ddots & & \\
& & & & -1 & 2 & -1 & \\
& & & & & -1 & 2 & -1 \\
& & & & & & -2 & 2
\end{array}\right) .
$$

With our choice of simple roots, we get the Borel subgroup $B=T N$, where $N$ consists of matrices of the form $\left(\begin{array}{cc}A & 0 \\ 0 & { }^{t} A^{-1}\end{array}\right)\left(\begin{array}{ll}1 & B \\ 0 & 1\end{array}\right)$, with $A \in \mathrm{GL}(n)$ lower triangular unipotent and $B$ symmetric. (Note that some authors use a different set of simple roots that results in $A$ being upper triangular as was pointed out by the referee.) The torus $T$ acts on the Lie-algebra $\mathfrak{n}$ of $N$ by the adjoint representation Ad. It is easy to compute the modular factor $\delta_{B}(t)=\operatorname{det}\left(\operatorname{Ad}_{\mathfrak{n}}(t)\right)$. If $t$ is given in the form (3), then

$$
\delta_{B}(t)=u_{0}^{-n(n+1) / 2} u_{1}^{2} u_{2}^{4} \cdot \ldots \cdot u_{n}^{2 n} .
$$




\section{Local representations}

In Theorem 2 we shall describe the local components of an automorphic representation associated with a classical Siegel modular form. This is done in terms of Satake parameters at the finite places, and in terms of the weight (or HarishChandra parameters, if the weight is large enough) at the archimedean place. In the following sections we shall therefore collect all the required facts about local representations.

Notation. In Sects. 3.1 through $3.4, F$ is a p-adic field, $\mathcal{O}$ its ring of integers, $\omega \in \mathcal{O}$ a generator of the maximal ideal, and $q=\sharp \mathcal{O} / \omega \mathcal{O}$. The symbols $G, T, \ldots$ denote $F$-points of the underlying algebraic groups. We let $K=\operatorname{GSp}(2 n, \mathcal{O})$.

Section 3.5 deals with the archimedean place, i.e., the underlying local field is $\mathbb{R}$.

\subsection{The Satake isomorphism for $\mathrm{GSp}(2 n)$}

The Satake isomorphism for a reductive $\mathfrak{p}$-adic group is nicely described in [Ca]. Let $\mathcal{H}(G, K)$ be the unramified Hecke algebra of $G$, consisting of compactly supported functions $f: G \rightarrow \mathbb{C}$ which are left and right $K$-invariant. The product in $\mathcal{H}(G, K)$ is given by convolution

$$
(f * g)(x)=\int_{G} f(x y) g\left(y^{-1}\right) d y .
$$

Let $^{\circ} T:=T(\mathcal{O})$. Then we also have the Hecke algebra $\mathcal{H}\left(T,{ }^{\circ} T\right)$. Special elements in this Hecke algebra are

$$
\begin{aligned}
X_{0} & :=\operatorname{char}\left(\operatorname{diag}\left(\mathcal{O}^{*}, \ldots, \mathcal{O}^{*}, \omega \mathcal{O}^{*}, \ldots, \omega \mathcal{O}^{*}\right)\right), \\
X_{1} & :=\operatorname{char}\left(\operatorname{diag}\left(\omega \mathcal{O}^{*}, \mathcal{O}^{*}, \ldots, \mathcal{O}^{*}, \omega^{-1} \mathcal{O}^{*}, \mathcal{O}^{*}, \ldots, \mathcal{O}^{*}\right)\right), \\
& \vdots \\
X_{n} & :=\operatorname{char}\left(\operatorname{diag}\left(\mathcal{O}^{*}, \ldots, \mathcal{O}^{*}, \omega \mathcal{O}^{*}, \mathcal{O}^{*}, \ldots, \mathcal{O}^{*}, \omega^{-1} \mathcal{O}^{*}\right)\right),
\end{aligned}
$$

where "char" stands for characteristic function. It is easily seen that

$$
X_{0}^{k}=\operatorname{char}\left(\operatorname{diag}\left(\mathcal{O}^{*}, \ldots, \mathcal{O}^{*}, \omega^{k} \mathcal{O}^{*}, \ldots, \omega^{k} \mathcal{O}^{*}\right)\right), \quad k \in \mathbb{Z},
$$

and similarly for the other $X_{i}$. It is then clear that

$$
\mathcal{H}\left(T,{ }^{\circ} T\right)=\mathbb{C}\left[X_{0}^{ \pm 1}, X_{1}^{ \pm 1}, \ldots, X_{n}^{ \pm 1}\right] .
$$

Now, for an element $f \in \mathcal{H}(G, K)$, the Satake transform is defined by

$$
(S f)(t)=\left|\delta_{B}(t)\right|^{1 / 2} \int_{N} f(t n) d n=\left|\delta_{B}(t)\right|^{-1 / 2} \int_{N} f(n t) d n .
$$


$S f$ is an element of $\mathcal{H}\left(T,{ }^{\circ} T\right)$, and in fact $S$ defines an isomorphism

$$
S: \mathcal{H}(G, K) \stackrel{\sim}{\longrightarrow} \mathcal{H}\left(T,{ }^{\circ} T\right)^{W} .
$$

Here $W$ denotes the Weyl group of $G$, which acts naturally on the torus and on $\mathcal{H}\left(T,{ }^{\circ} T\right)$, and we take invariant elements.

Assume now that $f=\operatorname{char}(K M K)$ with $K M K=\bigsqcup M_{i} K$. Because of the Iwasawa decomposition $G=B K$ we may assume that

$$
M_{i}=\left(\begin{array}{cc}
A_{i} & B_{i} \\
0 & \omega^{d_{i 0}{ }^{t}} A_{i}^{-1}
\end{array}\right) \text { with } A_{i}=\left(\begin{array}{ccc}
\omega^{d_{i 1}} & & 0 \\
& \ddots & \\
& & \\
* & & \omega^{d_{i n}}
\end{array}\right) .
$$

Here $\omega$ is a prime element and the $d_{i j}$ are integers. Note that $d_{i 0}$ does not depend on $i$, since it equals the valuation of $\mu(M)$.

Lemma 1. With $f$ as above, we have

$$
S f=q^{\delta n(n+1) / 4} X_{0}^{\delta} \sum_{i} \prod_{j=1}^{n}\left(q^{-j} X_{j}\right)^{d_{i j}} .
$$

where $\delta$ is the valuation of $\mu(M)$.

Proof. We have to compute

$$
(S f)(t)=\left|\delta_{B}(t)\right|^{1 / 2} \sum_{i} \int_{N} \mathbf{1}_{M_{i} K}(t n) d n,
$$

where we may assume $t=\operatorname{diag}\left(\omega^{k_{1}}, \ldots, \omega^{k_{n}}, \omega^{-k_{1}+k_{0}}, \ldots, \omega^{-k_{n}+k_{0}}\right)$. Consider $\int_{N} \mathbf{1}_{M_{i} K}(t n) d n$ for fixed $i$. We have $t n \in M_{i} K$ if and only if $n \in t^{-1} M_{i} K$. It is clear that we can find such an $n$ only if $t^{-1} M_{i}$ has units on the diagonal, i.e., if $k_{j}=d_{i j}$ for all $j$. Assuming this is the case, then with $n^{\prime}:=t^{-1} M_{i} \in N$ our integral equals

$$
\int_{N} \mathbf{1}_{n^{\prime} K}(n) d n=\int_{N} \mathbf{1}_{K}(n) d n=1 .
$$

We have proved that

$$
\int_{N} \mathbf{1}_{M_{i} K}(t n) d n= \begin{cases}1 & \text { if } k_{j}=d_{i j} \text { for all } j=0,1, \ldots, n \\ 0 & \text { otherwise. }\end{cases}
$$

With the characteristic functions $X_{j}$ defined above, this may be written as

$$
\left(t \longmapsto \int_{N} \mathbf{1}_{M_{i} K}(t n) d n\right)=X_{0}^{d_{i 0}} X_{1}^{d_{i 1}} \cdot \ldots \cdot X_{n}^{d_{i n}} .
$$

It follows from (6) that

$$
\left|\delta_{B}(t)\right|^{1 / 2}=q^{k_{0} n(n+1) / 4} q^{-k_{1}-2 k_{2}-\ldots-n k_{n}} .
$$

Multiplying this by the function (8), we may replace $k_{j}$ by $d_{i j}$, and the assertion follows. 


\subsection{Spherical representations}

An irreducible admissible representation of $G$ is called spherical if it contains a non-zero vector fixed by $K$. All the spherical representations of $G$ are obtained as follows. Let $\chi_{0}, \ldots, \chi_{n}$ be unramified characters of $F^{*}$ (i.e., homomorphisms $F^{*} \rightarrow \mathbb{C}^{*}$ which are trivial on $\mathcal{O}^{*}$ ). They define an unramified character of the Borel subgroup $B=T N$ which is trivial on $N$ and which, on $T$, is given by

$$
t \longmapsto \chi_{0}\left(u_{0}\right) \chi_{1}\left(u_{1}\right) \cdot \ldots \cdot \chi_{n}\left(u_{n}\right),
$$

with $t \in T$ of the form (3). Normalized induction to $G$ yields a representation which has a unique spherical constituent. We denote this spherical representation by

$$
\pi\left(\chi_{0}, \chi_{1}, \ldots, \chi_{n}\right) \text {. }
$$

The isomorphism class of this representation depends only on the unramified characters modulo the action of the Weyl group. It is further known that each spherical representation is obtained in this way. Thus there is a bijection between unramified characters of $T$ modulo the action of the Weyl group, and isomorphism classes of spherical representations of $G$.

Each unramified character of $F^{*}$ is determined by its value on a prime element $\omega \in F$. This value may be any non-zero complex number. With the Satake parameters $b_{i}:=\chi_{i}(\omega)$, the character (9) is thus also determined by the vector $\left(b_{0}, b_{1}, \ldots, b_{n}\right) \in\left(\mathbb{C}^{*}\right)^{n+1}$. The Weyl group acts on this complex torus, and we see that unramified representations of $G$ are parameterized by the orbit space $\left(\mathbb{C}^{*}\right)^{n+1} / W$. In fact, the Satake isomorphism

$$
S: \mathcal{H}(G, K) \stackrel{\sim}{\longrightarrow} \mathbb{C}\left[X_{0}^{ \pm 1}, X_{1}^{ \pm 1}, \ldots, X_{n}^{ \pm 1}\right]^{W}
$$

identifies the Hecke algebra with the coordinate ring of $\left(\mathbb{C}^{*}\right)^{n+1} / W$. Each point $\left(b_{0}, \ldots, b_{n}\right) \in\left(\mathbb{C}^{*}\right)^{n+1} / W$ determines a character, i.e. an algebra homomorphism $\mathbb{C}\left[X_{0}^{ \pm 1}, \ldots, X_{n}^{ \pm 1}\right]^{W} \rightarrow \mathbb{C}$, by mapping $X_{i}$ to $b_{i}$. Via $S$ this also defines a character of $\mathcal{H}(G, K)$, which is nothing but the action of $\mathcal{H}(G, K)$ on the one-dimensional space of spherical vectors in $\pi\left(\chi_{0}, \ldots, \chi_{n}\right)$.

These well-known facts may be summarized in the following commutative diagram, in which all the maps are bijections:

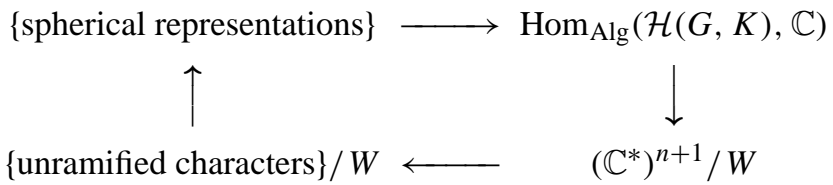

The left arrow is induction and then taking the spherical constituent, the top arrow is the action of $\mathcal{H}(G, K)$ on the space of spherical vectors, the map on the right comes from the identification $\mathcal{H}(G, K) \simeq \mathbb{C}\left[X_{0}^{ \pm 1}, \ldots, X_{n}^{ \pm 1}\right]^{W}$, and the bottom arrow assigns to the Satake parameters $\left(b_{0}, \ldots, b_{n}\right)$ the unramified character with $\chi_{i}(\omega)=b_{i}$. 
Lemma 1 can now be restated in the following way. Let $v$ be a spherical vector in $\pi:=\pi\left(\chi_{0}, \ldots, \chi_{n}\right)$, and let $f=\mathbf{1}_{K M K}$ be the element from Lemma 1 . Then

$$
\pi(f) v=q^{\delta n(n+1) / 4} b_{0}^{\delta} \sum_{i} \prod_{j=1}^{n}\left(q^{-j} b_{j}\right)^{d_{i j}} v, \quad \text { where } b_{j}=\chi_{j}(\omega) .
$$

Clearly the representation induced from the character (9) has central character $\chi_{0}^{2} \chi_{1} \ldots \chi_{n}$. This character is trivial if and only if the Satake parameters satisfy

$$
b_{0}^{2} b_{1} \cdot \ldots \cdot b_{n}=1 .
$$

Thus, exactly these parameters give spherical representations of the group $\operatorname{PGSp}(2 n, F)$.

\subsection{Dual groups}

In Sect. 2 we described the root datum

$$
\left(X, R, X^{\vee}, R^{\vee}\right)
$$

of $G=\operatorname{GSp}(2 n)$. By definition, the dual group $\hat{G}=\operatorname{GSpin}(2 n+1, \mathbb{C})$ has root datum

$$
\left(X^{\prime}, R^{\prime}, X^{\prime \vee}, R^{\prime \vee}\right)=\left(X^{\vee}, R^{\vee}, X, R\right) .
$$

Let $e_{0}^{\prime}, \ldots, e_{n}^{\prime}$ be a basis of $X^{\prime}$ such that

$$
e_{i}^{\prime}=f_{i}
$$

under the identification $X^{\prime}=X^{\vee}$, and let $f_{0}^{\prime}, \ldots, f_{n}^{\prime}$ be a basis of $X^{\prime \vee}$ such that

$$
f_{i}^{\prime}=e_{i}
$$

under the identification $X^{\prime \vee}=X$. The complex torus $\left(\mathbb{C}^{*}\right)^{n+1}$ of the previous section may be viewed as the maximal torus $\hat{T} \subset \hat{G}$. Elements of $\hat{T} / W$ are in one-one correspondence with semisimple conjugacy classes in $\hat{G}$. By the previous section, we have a correspondence between spherical representations of $G$ and those semisimple conjugacy classes. If $\chi$ is an unramified character of $T$ defining a spherical representation, and $\hat{t} \in \hat{T}$ is the associated parameter, then $\chi$ and $\hat{t}$ are related by the general relation

$$
\chi(\varphi(\omega))=\varphi(\hat{t}) \quad \text { for all } \varphi \in X^{\vee}=X^{\prime}
$$

(see [GS] p. 26). It is clear that each $\hat{t} \in \hat{T}$ can be written uniquely as

$$
\hat{t}=\prod_{i=0}^{n} f_{i}^{\prime}\left(t_{i}\right), \quad t_{i} \in \mathbb{C}^{*}
$$

We have the pairing $\left\langle e_{i}^{\prime}, f_{j}^{\prime}\right\rangle=\delta_{i j}$, and thus $t_{i}=e_{i}^{\prime}(\hat{t})$. Therefore putting $\varphi=e_{i}^{\prime}=$ $f_{i}$ in (12) yields $b_{i}=t_{i}$. We proved the following. 
Lemma 2. Let $\pi=\pi(\chi)$ be a spherical principal series representation of $G=$ $\operatorname{GSp}(2 n, F)$. Let $b_{0}, b_{1}, \ldots, b_{n}$ be the Satake parameters of $\pi$, as in 3.2. Then the associated semisimple conjugacy class in $\mathrm{GSpin}(2 n+1, \mathbb{C})$ is represented by

$$
\hat{t}=\prod_{i=0}^{n} f_{i}^{\prime}\left(b_{i}\right)
$$

Set $\bar{G}:=\operatorname{PGSp}(2 n, F)=G / C$, where $C$ is the center of $G$. The dual group of $\bar{G}$ is

$$
\hat{\bar{G}}=\operatorname{Spin}(2 n+1, \mathbb{C}) \subset \mathrm{GSpin}(2 n+1, \mathbb{C}),
$$

which is also the derived group of $\hat{G}$. The element $t \in \hat{T}$ will correspond to a representation of $G$ with trivial central character, i.e., to a representation of $\bar{G}$, if and only if it lies in

$$
\hat{\bar{T}}:=\hat{T} \cap \operatorname{Spin}(2 n+1, \mathbb{C}),
$$

the maximal torus of $\operatorname{Spin}(2 n+1, \mathbb{C})$.

\subsection{Local Euler factors}

For a spherical representation $\pi$ of $\bar{G}$ with parameter $\hat{t} \in \hat{\bar{T}}$ and a finite-dimensional representation

$$
\varrho: \operatorname{Spin}(2 n+1, \mathbb{C}) \longrightarrow \operatorname{GL}(m, \mathbb{C}),
$$

Langlands defines the local Euler factor $L(s, \pi, \varrho)=\operatorname{det}\left(\mathbf{1}-\varrho(\hat{t}) q^{-s}\right)^{-1}$. In this section we are going to compute this factor for the "projection representation"

$$
\varrho_{1}: \operatorname{Spin}(2 n+1, \mathbb{C}) \longrightarrow \operatorname{SO}(2 n+1, \mathbb{C}),
$$

and for the $2^{n}$-dimensional spin representation $\varrho_{2}$. Similar computations can also be found in $[\mathrm{As}]$.

The projection representation. One can find the weight structure of the representation $\varrho_{1}$ in $[\mathrm{FH}]$. All the weight spaces of this $(2 n+1)$-dimensional representation are one-dimensional. In the notation of the previous section the weights are

$$
e_{1}^{\prime}, \ldots, e_{n}^{\prime}, 0,-e_{1}^{\prime}, \ldots,-e_{n}^{\prime} .
$$

The eigenvalues of an operator $\rho(\hat{t})$ are therefore 1 and

$$
e_{i}^{\prime}(\hat{t})^{ \pm 1}, \quad i=1, \ldots, n .
$$

With $\hat{t}$ as in Lemma 2, we get $e_{i}^{\prime}(\hat{t})=b_{i}$. This proves the following.

Lemma 3. If $\pi$ is a spherical representation of $\bar{G}$ with Satake parameters $b_{0}, b_{1}, \ldots, b_{n}$ as in 3.2, then

$$
L\left(s, \pi, \varrho_{1}\right)^{-1}=\left(1-q^{-s}\right) \prod_{i=1}^{n}\left(1-b_{i} q^{-s}\right)\left(1-b_{i}^{-1} q^{-s}\right) .
$$


The spin representation. Again from $[\mathrm{FH}]$, the $2^{n}$-dimensional spin representation $\varrho_{2}$ of $\operatorname{Spin}(2 n+1, \mathbb{C})$ has highest weight $\frac{1}{2}\left(e_{1}^{\prime}+\ldots+e_{n}^{\prime}\right)$. Indeed, all the weight spaces are one-dimensional, and the weights of $\varrho_{2}$ are

$$
\frac{c_{1} e_{1}^{\prime}+\ldots+c_{n} e_{n}^{\prime}}{2}, \quad c_{i} \in\{ \pm 1\} .
$$

Let such a weight be given. Define a character $\eta$ of $\hat{T}$ by

$$
\eta(\hat{t})=t_{0} \prod_{\substack{i=1 \\ c_{i}=1}}^{n} t_{i}, \quad \text { if } \hat{t}=\prod_{i=0}^{n} f_{i}^{\prime}\left(t_{i}\right)
$$

We claim that $\eta$ coincides with the character (15) on $\hat{\bar{T}}$, the maximal torus of $\operatorname{Spin}(2 n+1, \mathbb{C})$. An element $\hat{t}$ as above lies in this maximal torus if and only if $t_{0}^{2} t_{1} \cdot \ldots \cdot t_{n}=1$. Assuming this relation, we have

$$
\begin{aligned}
\left(c_{1} e_{1}^{\prime}+\ldots+c_{n} e_{n}^{\prime}\right)(\hat{t}) & =\prod_{i=1}^{n}\left(e_{i}^{\prime}(\hat{t})\right)^{c_{i}}=\prod_{i=1}^{n} t_{i}^{c_{i}} \\
& =t_{0}^{2} \prod_{i=1}^{n} t_{i}^{c_{i}+1}=t_{0}^{2} \prod_{\substack{i=1 \\
c_{i}=1}}^{n} t_{i}^{2}=(2 \eta)(\hat{t}),
\end{aligned}
$$

and it follows that indeed $\frac{1}{2}\left(c_{1} e_{1}^{\prime}+\ldots+c_{n} e_{n}^{\prime}\right)=\eta$ on $\hat{\bar{T}}$. We can thus easily compute the eigenvalues of $\varrho_{2}(\hat{t})$, and get the following lemma.

Lemma 4. If $\pi$ is a spherical representation of $\bar{G}$ with Satake parameters $b_{0}, b_{1}, \ldots, b_{n}$ as in 3.2, then

$$
L\left(s, \pi, \varrho_{2}\right)^{-1}=\prod_{k=0}^{n} \prod_{1 \leq i_{1}<\ldots<i_{k} \leq n}\left(1-b_{0} b_{i_{1}} \ldots b_{i_{k}} q^{-s}\right) .
$$

\subsection{Lowest weight representations for $\operatorname{PGSp}(2 n, \mathbb{R})$}

In this section we shall construct a class of lowest weight representations of $\operatorname{PGSp}(2 n, \mathbb{R})$, which appear as the infinite component in the automorphic representation attached to a holomorphic Siegel modular form.

The Lie algebra of $\operatorname{Sp}(2 n, \mathbb{R})$ is explicitly given by

$$
\begin{aligned}
\mathfrak{g} & =\left\{X \in M(2 n, \mathbb{R}): X J+J^{t} X=0\right\} \\
& =\left\{\left(\begin{array}{ll}
A & B \\
C & D
\end{array}\right) \in M(2 n, \mathbb{R}): B={ }^{t} B, C={ }^{t} C, A=-{ }^{t} D\right\} .
\end{aligned}
$$

The standard maximal compact subgroup $K_{\infty}$ of $\operatorname{Sp}(2 n, \mathbb{R})$ is

$$
K_{\infty}=\left\{\left(\begin{array}{cc}
A & B \\
-B & A
\end{array}\right) \in \operatorname{GL}(2 n, \mathbb{R}): A^{t} A+B{ }^{t} B=\mathbf{1}, A^{t} B=B^{t} A\right\} .
$$


We have $K_{\infty} \simeq U(n)$ via $\left(\begin{array}{cc}A & B \\ -B & A\end{array}\right) \longmapsto A+i B$. The Lie algebra of $K_{\infty}$ is

$$
\mathfrak{k}=\left\{\left(\begin{array}{cc}
A & B \\
-B & A
\end{array}\right) \in M(2 n, \mathbb{R}): A=-{ }^{t} A, B={ }^{t} B\right\} .
$$

This is also the 1-eigenspace of the Cartan involution $\theta X=-{ }^{t} X$. The $(-1)-$ eigenspace is

$$
\mathfrak{p}=\left\{\left(\begin{array}{cc}
A & B \\
B & -A
\end{array}\right) \in M(2 n, \mathbb{R}): A={ }^{t} A, B={ }^{t} B\right\},
$$

so that $\mathfrak{g}=\mathfrak{k} \oplus \mathfrak{p}$. It is easy to see that $\mathfrak{p}_{\mathbb{C}}=\mathfrak{p}_{\mathbb{C}}^{+} \oplus \mathfrak{p}_{\mathbb{C}}^{-}$, with

$$
\mathfrak{p}_{\mathbb{C}}^{ \pm}=\left\{\left(\begin{array}{cc}
A & \pm i A \\
\pm i A & -A
\end{array}\right) \in M(2 n, \mathbb{C}): A={ }^{t} A\right\} .
$$

Each of $\mathfrak{p}_{\mathbb{C}}^{ \pm}$is an abelian subalgebra of $\mathfrak{g}_{\mathbb{C}}$, we have $\left[\mathfrak{k}_{\mathbb{C}}, \mathfrak{p}_{\mathbb{C}}^{ \pm}\right] \subset \mathfrak{p}_{\mathbb{C}}^{ \pm}$, and

$$
\mathfrak{g}_{\mathbb{C}}=\mathfrak{p}_{\mathbb{C}}^{+} \oplus \mathfrak{k}_{\mathbb{C}} \oplus \mathfrak{p}_{\mathbb{C}}^{-}
$$

In the complexified Lie-algebra $\mathfrak{k}_{\mathbb{C}}$ of $K$ let

$$
T_{i}=-i\left(\begin{array}{cc}
0 & D_{i} \\
-D_{i} & 0
\end{array}\right)
$$

where $D_{i}$ is the diagonal matrix with entry 1 at position $(i, i)$, and zeros elsewhere. Then $\mathfrak{h}=\mathbb{R} T_{1}+\ldots+\mathbb{R} T_{n}$ is a compact Cartan subalgebra of $\mathfrak{g}_{\mathbb{C}}$. Let $e_{i}$ be the linear form on $\mathfrak{h}_{\mathbb{C}}$ which sends $T_{i}$ to 1 , and $T_{j}$ to 0 for $j \neq i$. Then the following is a system of positive roots for $\left(\mathfrak{g}_{\mathbb{C}}, \mathfrak{h}_{\mathbb{C}}\right)$ :

$$
\begin{array}{ll}
2 e_{j}, & 1 \leq j \leq n, \\
e_{j}+e_{k}, & 1 \leq j<k \leq n, \\
e_{j}-e_{k}, & 1 \leq j<k \leq n
\end{array}
$$

(the simple roots are $e_{1}-e_{2}, \ldots, e_{n-1}-e_{n}, 2 e_{n}$ ). In fact, $\mathfrak{p}_{\mathbb{C}}^{+}$is spanned by the root spaces for the first two types of roots. If $D_{j}$ is as above, then

$$
\left(\begin{array}{cc}
D_{j} & i D_{j} \\
i D_{j} & -D_{j}
\end{array}\right), \quad 1 \leq j \leq n
$$

spans the root space for the root $2 e_{j}$, and if $E_{j k}$ is the matrix with entry 1 at positions $(j, k)$ and $(k, j)$, and zeros elsewhere, then

$$
\left(\begin{array}{cc}
E_{j k} & i E_{j k} \\
i E_{j k} & -E_{j k}
\end{array}\right), \quad 1 \leq j<k \leq n,
$$

spans the root space for the root $e_{j}+e_{k}$. These roots are the non-compact positive roots. The other positive roots are compact; the root space of $e_{j}-e_{k}$ is spanned by the element

$$
\left(\begin{array}{cc}
F_{j k} & -i E_{j k} \\
i E_{j k} & F_{j k}
\end{array}\right), \quad 1 \leq j<k \leq n
$$


where $F_{j k}$ is the matrix with entry 1 at position $(j, k)$, entry -1 at position $(k, j)$, and zeros elsewhere.

Let $k$ be a positive integer (it will be the weight of a Siegel modular form in our case). We shall construct a lowest weight representation of $G$ with lowest weight $k\left(e_{1}+\ldots+e_{n}\right)$. Let $\Xi_{k}$ be the character of $K$ given by

$$
\Xi_{k}(\kappa)=j(\kappa, I)^{-k},
$$

where $j(g, Z)=\operatorname{det}(C Z+D)$ is the classical automorphic factor, $g=\left(\begin{array}{ll}A & B \\ C & D\end{array}\right) \epsilon$ $G$, and $Z$ is in the Siegel upper half plane ( $\Xi$ is a character because $K$ is the stabilizer of $I=\operatorname{diag}(i, \ldots, i)$ under linear fractional transformations). Let $\xi_{k}=d \Xi_{k}$ be the differential, complexified to a linear form $\mathfrak{k}_{\mathbb{C}} \rightarrow \mathbb{C}$. Explicitly,

$$
\begin{array}{ll}
\xi_{k}\left(T_{j}\right)=k, & j=1, \ldots, n, \\
\xi_{k}\left(X_{e_{j}-e_{l}}\right)=0 & \text { for all } j \neq l .
\end{array}
$$

Because of $\left[\mathfrak{k}_{\mathbb{C}}, \mathfrak{p}_{\mathbb{C}}^{-}\right] \subset \mathfrak{p}_{\mathbb{C}}^{-}$, the character $\xi_{k}$ can be extended to a character of $\mathfrak{k}_{C}+\mathfrak{p}_{\mathbb{C}}^{-} \cdot$ Consider

$$
V_{k}=\mathcal{U}\left(\mathfrak{g}_{\mathbb{C}}\right) \otimes_{\mathcal{U}\left(\mathfrak{k}_{\mathbb{C}}+\mathfrak{p}_{\mathbb{C}}^{-}\right)} \mathbb{C}_{\xi_{k}}
$$

Here $\mathbb{C}_{\xi_{k}}=\mathbb{C}$ with the action of $\mathfrak{k}_{\mathbb{C}}+\mathfrak{p}_{\mathbb{C}}^{-}$given by $\xi_{k}$. If $v_{0}$ denotes the special vector $1 \otimes 1 \in V_{k}$, then $T_{j} v_{0}=k v_{0}$, and $v_{0}$ is annihilated by all negative roots. Thus we have a representation of $\mathfrak{g}_{\mathbb{C}}$ of lowest weight $k\left(e_{1}+\ldots+e_{n}\right)$. It is also clear that

$$
V_{k}=\mathcal{U}\left(\mathfrak{p}_{\mathbb{C}}^{+}\right) v_{0}
$$

$V_{k}$ is in fact a $\left(\mathfrak{g}_{\mathbb{C}}, K\right)$-module, and globalizes to a unitary representation of $\operatorname{Sp}(2 n, \mathbb{R})$ which in the following we shall denote by $\pi_{k}$ (it depends only on the positive integer $k)$. The center of $\operatorname{Sp}(2 n, \mathbb{R})$ consists of only two elements, and it is easy to see that the central character of $\pi_{k}$ is given by $(-1)^{n k}$. Thus, if $n k$ is even, $\pi_{k}$ descends to a representation of $\operatorname{PSp}(2 n, \mathbb{R})$. Assuming this is the case, we get a representation of $\operatorname{PGSp}(2 n, \mathbb{R})$ by inducing $\pi_{k}$ from the $\operatorname{subgroup} \operatorname{PSp}(2 n, \mathbb{R})$ (of index 2). This new representation shall also be denoted by $\pi_{k}$. It has a lowest weight vector of weight $k\left(e_{1}+\ldots+e_{n}\right)$, and a highest weight vector of weight $-k\left(e_{1}+\ldots+e_{n}\right)$.

By Harish-Chandra, the discrete series representations of $\operatorname{Sp}(2 n, \mathbb{R})$ are parameterized by elements in the weight lattice which do not lie on a wall, modulo the action of the Weyl group of $K$ (see, for instance, [Kna] Theorem 9.20). If $\lambda$ is such a Harish-Chandra parameter, then the lowest $K$-type of the corresponding discrete series representation is given by the Blattner parameter

$$
\Lambda=\lambda+\delta_{\mathrm{nc}}-\delta_{\mathrm{c}},
$$

where $\delta_{\text {nc }}$ (resp. $\delta_{\mathrm{c}}$ ) is half of the sum of the non-compact (resp. compact) positive roots; here "positive" means with respect to the Weyl chamber in which $\lambda$ lies. 
The choice (20) of positive roots leads to the holomorphic discrete series representations. The corresponding Harish-Chandra parameters are of the form

$$
\lambda=a_{1} e_{1}+\ldots+a_{n} e_{n}, \quad a_{i} \in \mathbb{Z}, a_{1}>\ldots>a_{n}>0 .
$$

The relation with the Blattner parameter is

$$
\Lambda=\lambda+\sum_{j=1}^{n} j e_{j} .
$$

We shall be particularly interested in the discrete series representation with HarishChandra parameter

$$
(k-1) e_{1}+\ldots(k-n) e_{n},
$$

where $k>n$ is an integer; it has the lowest $K$-type $k e_{1}+\ldots+k e_{n}$, and must thus coincide with the representation $\pi_{k}$ constructed above. If $k=n$, then the HarishChandra parameter comes to lie on a wall and this identifies our $\pi_{k}, k=n$, as a limit of discrete series. If $k<n$, then $\pi_{k}$ is neither a discrete series representation, nor a limit of them.

\section{Siegel modular forms}

For basic facts about classical Siegel modular forms we refer to [AZ], [Fr1], or $[\mathrm{K} 1]$.

\subsection{Lifting of Siegel modular forms}

Siegel modular forms of degree $n$ are certain holomorphic functions on the Siegel upper half plane $\mathbb{H}_{n}$, which by definition is the complex manifold consisting of complex symmetric $n \times n$ matrices with positive definite imaginary part. Strong approximation allows one to regard Siegel modular forms of degree $n$ as functions on $G(\mathbb{A})$, where $G=\mathrm{GSp}(2 n)$, and where the ground field is $\mathbb{Q}$.

Let $f$ be a Siegel modular form of weight $k$ and degree $n$. We shall assume that $f$ is a modular form with respect to the full modular group $\Gamma_{n}=\operatorname{Sp}(2 n, \mathbb{Z})$, i.e., $\left.f\right|_{k} \gamma=f$ for all $\gamma \in \Gamma_{n}$, where

$$
\left(\left.f\right|_{k} h\right)(Z)=\mu(h)^{n k / 2} j(h, Z)^{-k} f(h\langle Z\rangle) \text { for } h \in G_{\infty}^{+}, Z \in \mathbb{H}_{n}
$$

(here $\mu$ is the multiplier, $j(h, Z)=\operatorname{det}(C Z+D)$ for $h=\left(\begin{array}{ll}A & B \\ C & D\end{array}\right)$, and $h\langle Z\rangle=$ $\left.(A Z+B)(C Z+D)^{-1}\right)$. We remark that this operation differs from the classical one used in $[\mathrm{An}]$ by a factor. We do so to make the center of $G(\mathbb{A})$ act trivially. More precisely, the classical operation is

$$
\left(f \|_{k} h\right)(Z)=\operatorname{det}(h)^{k-(n+1) / 2} j(h, Z)^{-k} f(h\langle Z\rangle) .
$$


The relation between the two operations is

$$
f \|_{k} h=\left.\operatorname{det}(h)^{(k-n-1) / 2} f\right|_{k} h \quad \text { for all } h \in G_{\infty}^{+} .
$$

To $f$ is associated a function $\Phi_{f}: G(\mathbb{A}) \rightarrow \mathbb{C}$ as follows. One uses strong approximation for $\operatorname{Sp}(2 n)$ (cf. [Kne]) to show that

$$
G(\mathbb{A})=G(\mathbb{Q}) G(\mathbb{R})^{+} \prod_{p<\infty} G\left(\mathbb{Z}_{p}\right),
$$

where $G(\mathbb{R})^{+}$denotes those elements of $G(\mathbb{R})$ which have positive multiplier. Write an element $g \in G(\mathbb{A})$ as

$$
g=g_{\mathbb{Q}} g_{\infty} k_{0} \quad \text { with } g_{\mathbb{Q}} \in G(\mathbb{Q}), g_{\infty} \in G_{\infty}^{+}, k_{0} \in K_{0},
$$

where $K_{0}=\prod_{p<\infty} K_{p}$ with $K_{p}=G\left(\mathbb{Z}_{p}\right)$. Then we define

$$
\Phi_{f}(g)=\left(\left.f\right|_{k} g_{\infty}\right)(I)
$$

where $I=\operatorname{diag}(i, \ldots, i) \in \mathbb{H}_{n}$. This is well-defined because of the transformation properties of $f$.

The map $f \mapsto \Phi_{f}$ injects the space of modular forms of weight $k$ into a space of functions $\Phi$ on $G_{\mathbb{A}}$ having the following properties:

i) $\Phi(\varrho g)=\Phi(g)$ for $\varrho \in G(\mathbb{Q})$,

ii) $\Phi\left(g k_{0}\right)=\Phi(g)$ for $k_{0} \in K_{0}$,

iii) $\Phi\left(g k_{\infty}\right)=\Phi(g) j\left(k_{\infty}, I\right)^{-k}$ for $k_{\infty} \in K_{\infty}$,

iv) $\Phi(g z)=\Phi(g)$ for $z \in Z(\mathbb{A})$.

Here $Z \simeq \mathrm{GL}(1)$ is the center of $\mathrm{GSp}(2 n)$, and $K_{\infty} \simeq U(n)$ is the standard maximal compact subgroup of $\operatorname{Sp}(2 n, \mathbb{R}$ ) (and is equal to the stabilizer of $I$ under linear fractional transformations; see also the next section).

Lemma 5. If $f \in S_{k}\left(\Gamma_{n}\right)$, then the automorphic form $\Phi_{f}$ is cuspidal, i.e.,

$$
\int_{N(\mathbb{Q}) \backslash N(\mathbb{A})} \Phi_{f}(n g) d n=0 \quad \text { for all } g \in G(\mathbb{A})
$$

for each unipotent radical $N$ of each proper parabolic subgroup of $G$.

Proof. It is enough to verify the cusp condition for the standard maximal parabolics. If $P=M N$ is one of those, then by the Iwasawa decomposition we may assume $g \in M(\mathbb{A})$. Using strong approximation for $M(\mathbb{A})$, we may further assume that $g \in M_{\infty}^{+}:=M(\mathbb{R}) \cap G_{\infty}^{+}$. Let $V$ be the intersection of the unipotent radicals of all standard maximal parabolics. Clearly it is enough to show that

$$
\int_{V(\mathbb{Q}) \backslash V(\mathbb{A})} \Phi_{f}(n g) d n=0 .
$$


Now $V$ consists of all elements of the form $\left(\begin{array}{rr}1 & v \\ 1\end{array}\right)$ with $v \in V^{\prime}$, where $V^{\prime}$ is the set of symmetric $n \times n$ matrices with non-zero entries only in last row and last column (thus $V \simeq V^{\prime} \simeq \mathbb{G}_{a}^{n}$ ). This is easy to see from the fact that a root space $X_{\alpha}$ belongs to $V$ if and only if $\alpha=c_{1} \alpha_{1}+\ldots+c_{n} \alpha_{n}$ with all $c_{i}>0$ (and $\alpha_{i}$ as in (4)). With $Z:=g\langle I\rangle$ one gets

$$
\begin{aligned}
& \int_{V(\mathbb{Q}) \backslash V(\mathbb{A})} \Phi_{f}(n g) d n=\int_{V(\mathbb{Z}) \backslash V(\mathbb{R})} \Phi_{f}(n g) d n \\
= & \int_{V(\mathbb{Z}) \backslash V(\mathbb{R})} f(n g\langle I\rangle) j(n g, I)^{-k} d n=\int_{V^{\prime}(\mathbb{Z}) \backslash V^{\prime}(\mathbb{R})} f(Z+v) j(g, I)^{-k} d v .
\end{aligned}
$$

At this point we use the Fourier expansion of $f$ :

$$
f(Z)=\sum_{R} c_{R} \exp (2 \pi i \operatorname{tr}(R Z)), \quad Z \in \mathbb{H}_{n},
$$

where $R$ runs over semi-integral, positive definite matrices (it is here where we use the classical cusp condition). Thus

$$
\begin{gathered}
\int_{V(\mathbb{Q}) \backslash V(\mathbb{A})} \Phi_{f}(n g) d n=\sum_{R} c_{R} \exp (2 \pi i \operatorname{tr}(R Z)) j(g, I)^{-k} \\
\cdot \int_{V^{\prime}(\mathbb{Z}) \backslash V^{\prime}(\mathbb{R})} \exp (2 \pi i \operatorname{tr}(R v)) d v .
\end{gathered}
$$

Using the fact that $R$ is non-degenerate, one checks that the map

$$
v \mapsto \exp (2 \pi i \operatorname{tr}(R v))
$$

is a non-trivial character of $V^{\prime}$. Thus our integral is zero.

The lemma shows that the map $f \mapsto \Phi_{f}$ gives a one-one correspondence between the space $S_{k}\left(\Gamma_{n}\right)$ of cusp forms of weight $k$, and a subspace of $L_{0}^{2}(Z(\mathbb{A}) G(\mathbb{Q}) \backslash G(\mathbb{A}))$, the space of cuspidal functions in

$$
L^{2}(Z(\mathbb{A}) G(\mathbb{Q}) \backslash G(\mathbb{A})) .
$$

Also note that

$$
\Gamma_{n} \backslash \mathbb{H}_{n} \simeq Z(\mathbb{A}) G(\mathbb{Q}) \backslash G(\mathbb{A}) / K .
$$

To see this, map an element $g=g_{\mathbb{Q}} g_{\infty} k \in G(\mathbb{A})$ to $g_{\infty}\langle I\rangle$. Also, every point of the left hand side of the isomorphism can be written as $g_{\infty}\langle I\rangle$ for some $g_{\infty} \in G_{\infty}^{+}$ which can in turn be mapped to the image of $g_{\infty}$ in the right hand side.

If we start with a Haar measure on $G(\mathbb{A})$ and take the induced measure on the right side of (24), then the corresponding measure on the left side is induced from (a suitable multiple of) the usual invariant volume element $d^{*} Z$ on $\mathbb{H}_{n}$. In other 
words, if a $\Gamma_{n}$-invariant function $f$ on $\mathbb{H}_{n}$ and a $G(\mathbb{Q})$-invariant function $\Phi$ on $Z(\mathbb{A}) \backslash G(\mathbb{A}) / K$ are related by

$$
f\left(g_{\infty}\langle I\rangle\right)=\Phi\left(g_{\infty}\right) \quad \text { for all } g_{\infty} \in G_{\infty}^{+}
$$

then

$$
\int_{\Gamma_{n} \backslash \mathbb{H}_{n}} f(Z) d^{*} Z=\int_{Z(\mathbb{A}) G(\mathbb{Q}) \backslash G(\mathbb{A}) / K} \Phi(g) d g=\int_{Z(\mathbb{A}) G(\mathbb{Q}) \backslash G(\mathbb{A})} \Phi(g) d g
$$

(integrations taken over fundamental domains). We apply this to the function

$$
\begin{aligned}
\Phi_{f_{1}}(g) \overline{\Phi_{f_{2}}(g)} & =f_{1}(g\langle I\rangle) \overline{f_{2}(g\langle I\rangle)} \mu(g)^{n k}|j(g, I)|^{-2 k} \\
& =f_{1}(Z) \overline{f_{2}(Z)} \operatorname{det}(Y)^{k}
\end{aligned}
$$

where $f_{1}, f_{2} \in S_{k}\left(\Gamma_{n}\right)$, and where $g\langle I\rangle=X+i Y$ for $g \in G_{\infty}^{+}$. We obtain

$$
\int_{\Gamma_{n} \backslash \mathbb{H}_{n}} f_{1}(Z) \overline{f_{2}(Z)} \operatorname{det}(Y)^{k} d^{*} Z=\int_{Z(\mathbb{A}) G(\mathbb{Q}) \backslash G(\mathbb{A})} \Phi_{f_{1}}(g) \overline{\Phi_{f_{2}}(g)} d g .
$$

On the left, we have the classical Petersson scalar product, and the ordinary $L^{2}$ scalar product on the right. This proves the following.

Lemma 6. Upon suitable normalization of measures, the map $f \mapsto \Phi_{f}$ from $S_{k}\left(\Gamma_{n}\right)$ into $L_{0}^{2}(Z(\mathbb{A}) G(\mathbb{Q}) \backslash G(\mathbb{A}))$ is an isometry.

\subsection{Holomorphy and differential operators}

We are going to express the holomorphy of the function $f$ on $\mathbb{H}_{n}$ in terms of the annihilation of $\Phi_{f}$ by certain differential operators. If $\Phi$ is a function on $G(\mathbb{A})$ which is smooth as a function of $G(\mathbb{R})$, and if $X \in \mathfrak{g}$, the real Lie-algebra of $\operatorname{Sp}(2 n, \mathbb{R})$, then we define as usual

$$
(X \Phi)(g):=\left.\frac{d}{d t}\right|_{0} \Phi(g \exp (t X)), \quad g \in G(\mathbb{A}) .
$$

This action of $\mathfrak{g}$ on smooth functions $G(\mathbb{A}) \rightarrow \mathbb{C}$ extends to an action of $\mathfrak{g} \mathbb{C}$ by linearity. We have identified $\mathfrak{g}$ with a space of matrices in (16).

The stabilizer of $I \in \mathbb{H}_{n}$ (under linear fractional transformations) is the maximal compact subgroup $K_{\infty} \simeq U(n)$ of $\operatorname{Sp}(2 n, \mathbb{R})$, given by (17). As in 3.5 we have $\mathfrak{g}=\mathfrak{k} \oplus \mathfrak{p}$, where $\mathfrak{k}$ is the Lie algebra of $K$, and $\mathfrak{p}$ is the $(-1)$-eigenspace under the Cartan involution. Consider the projection

$$
\begin{aligned}
\rho: \operatorname{Sp}(2 n, \mathbb{R}) & \longrightarrow \mathbb{H}_{n}, \\
g & \longmapsto g\langle I\rangle,
\end{aligned}
$$


which induces the homeomorphism $\operatorname{Sp}(2 n, \mathbb{R}) / K_{\infty} \simeq \mathbb{H}_{n}$. Its differential at the identity

$$
d \rho: \mathfrak{g} \longrightarrow T_{I} \mathbb{H}_{n}
$$

has kernel $\mathfrak{k}$, and therefore induces an isomorphism $\mathfrak{p} \stackrel{\sim}{\longrightarrow} T_{I} \mathbb{H}_{n}$. If $T_{I} \mathbb{H}_{n}$ is naturally identified with the space of symmetric complex $n \times n$ matrices, then a small calculation shows that

$$
d \rho(X)=\left.\frac{d}{d t}\right|_{0} \exp (t X)\langle I\rangle=2(B+I A) \quad \text { for } X=\left(\begin{array}{cc}
A & B \\
B & -A
\end{array}\right) \in \mathfrak{p} .
$$

Let $J$ be the complex structure (multiplication with $i$ ) on $T_{I} \mathbb{H}_{n}$. Carried over to $\mathfrak{p}$, it is given by

$$
J\left(\begin{array}{cc}
A & B \\
B & -A
\end{array}\right)=\left(\begin{array}{cc}
B & -A \\
-A & -B
\end{array}\right)
$$

Now we have $\mathfrak{p}_{\mathbb{C}}=\mathfrak{p} \otimes_{\mathbb{R}} \mathbb{C}=\mathfrak{p}_{\mathbb{C}}^{+} \oplus \mathfrak{p}_{\mathbb{C}}^{-}$, where $\mathfrak{p}_{\mathbb{C}}^{ \pm}$is the $( \pm i)$-eigenspace of $J$. Thus the elements of $\mathfrak{p}_{\mathbb{C}}^{+}$(resp. $\mathfrak{p}_{\mathbb{C}}^{-}$) correspond to linear combinations of differential operators

$$
\frac{d}{d z_{j}} \in T_{I} \mathbb{H}_{n} \otimes_{\mathbb{R}} \mathbb{C} \quad\left(\text { resp. } \frac{d}{d \bar{z}_{j}}\right),
$$

where $z_{j}=x_{j}+i y_{j}$ are coordinates on $\mathbb{H}_{n}$ about the point $I$. Explicitly, we have

$$
\mathfrak{p}_{\mathbb{C}}^{ \pm}=\left\{\left(\begin{array}{cc}
A & \pm i A \\
\pm i A & -A
\end{array}\right) \in M(2 n, \mathbb{C}): A={ }^{t} A\right\},
$$

thus these spaces $\mathfrak{p}_{\mathbb{C}}^{ \pm}$coincide with the ones already defined in (18).

Lemma 7. Let $f$ be a smooth function on $\mathbb{H}_{n}$ transforming like a modular form, and let the function $\Phi_{f}$ on $G(\mathbb{A})$ be defined by (23). Then $f$ is holomorphic if and only if $\mathfrak{p}_{\mathbb{C}}^{-} \cdot \Phi_{f}=0$.

Proof. By the transformation properties of $\Phi$, it is enough to prove the following. Let $f$ be a smooth function on $\mathbb{H}_{n}$, and define $\Phi: \operatorname{Sp}(2 n, \mathbb{R}) \rightarrow \mathbb{C}$ by

$$
\Phi(g)=f(g\langle I\rangle) j(g, I)^{-k}, \quad g \in \operatorname{Sp}(2 n, \mathbb{R}) .
$$

Then $f$ is holomorphic if and only if $\mathfrak{p}_{\mathbb{C}}^{-} \cdot \Phi=0$.

Let $\tilde{f}(g)=f(g\langle I\rangle)$ and $\tilde{j}(g)=j(g, I)^{-k}$. We are going to prove that

$$
(X \Phi)(g)=(X \tilde{f})(g) j(g, I)^{-k} \quad \text { for } X \in \mathfrak{p}_{\mathbb{C}}^{-} .
$$

By considering the function $f^{\prime}(Z):=f(h\langle Z\rangle) j(h, Z)^{-k}$ instead of $f$, it is enough to do this for $g=\mathbf{1}$, i.e., we will show that

$$
(X \Phi)(\mathbf{1})=(X \tilde{f})(\mathbf{1}) \quad \text { for } X \in \mathfrak{p}_{\mathbb{C}}^{-}
$$


By the product rule, we have

$$
(X \Phi)(\mathbf{1})=(X \tilde{f})(\mathbf{1})+f(I)(X \tilde{j})(\mathbf{1}) \quad \text { for } X \in \mathfrak{g} \mathbb{C}
$$

(this is immediate for $X \in \mathfrak{g}$, and also holds for $X \in \mathfrak{g}_{\mathbb{C}}$ by linearity). Using (27), one gets $(X \tilde{j})(\mathbf{1})=0$ for $X \in \mathfrak{p}_{\mathbb{C}}^{-}$. This proves (29), and therefore also (28). Thus $\Phi$ is annihilated by $\mathfrak{p}_{\mathbb{C}}^{-}$if and only $\tilde{f}$ is. But by the definition of $\mathfrak{p}_{\mathbb{C}}^{-}$we have $\mathfrak{p}_{\mathbb{C}}^{-} \cdot \tilde{f}=0$ if and only if $f$ is holomorphic (see (26)).

\subsection{Hecke operators}

We now fix a prime number $p$. Let $\mathcal{H}_{p}^{\text {class }}$ be the $p$-component of the classical Hecke algebra of $G=\mathrm{GSp}(2 n)$. It is spanned by double cosets

$$
\Gamma M \Gamma \quad \text { with } M \in G\left(\mathbb{Z}\left[p^{-1}\right]\right)^{+},
$$

where $\mathbb{Z}\left[p^{-1}\right]$ is the ring of rational numbers with only $p$-powers in the denominator.

Lemma 8. There is an isomorphism

$$
\mathcal{H}_{p}^{\text {class }} \stackrel{\sim}{\longrightarrow} \mathcal{H}\left(G_{p}, K_{p}\right) .
$$

The double coset $\Gamma M \Gamma$ corresponds under this isomorphism to the characteristic function of $K_{p} M K_{p}$.

Proof. It is easy to see that there are bijections

$$
\Gamma \backslash G\left(\mathbb{Z}\left[p^{-1}\right]\right)^{+} / \Gamma \stackrel{\sim}{\longleftrightarrow} G(\mathbb{Z}) \backslash G\left(\mathbb{Z}\left[p^{-1}\right]\right) / G(\mathbb{Z}) \stackrel{\sim}{\longleftrightarrow} K_{p} \backslash G_{p} / K_{p},
$$

induced by the inclusions of the groups. Thus the Hecke algebras are isomorphic as vector spaces. There are similar bijections with cosets instead of double cosets. This fact is used to check that the classical multiplication of double cosets coincides with the convolution product on the $p$-adic Hecke algebra.

From now on we may identify the two Hecke algebras. We recall how these algebras act on modular forms. If $f$ is a classical modular form of weight $k$ and degree $n$, and if $T=\Gamma M \Gamma \in \mathcal{H}_{p}^{\text {class }}$, then

$$
\left.f\right|_{k} T:=\left.\sum_{i} f\right|_{k} M_{i}, \quad \text { where } \Gamma M \Gamma=\coprod_{i} \Gamma M_{i},
$$

is again a modular form. This defines a right action of $\mathcal{H}_{p}^{\text {class }}$ on the space of modular forms of weight $k$ and degree $n$. As before, this action differs slightly from Andrianov's action, which is defined with $\|_{k}$ instead of $\left.\right|_{k}$. It follows from (21) that

$$
f \|_{k} T=\left.\operatorname{det}(M)^{(k-n-1) / 2} f\right|_{k} T \text { for } T=\Gamma M \Gamma .
$$


There is also a left action of $\mathcal{H}\left(G_{p}, K_{p}\right)$ on adelic functions given by

$$
(T \Phi)(g)=\int_{G_{p}} T(h) \Phi(g h) d h, \quad T \in \mathcal{H}\left(G_{p}, K_{p}\right), g \in G(\mathbb{A}) .
$$

If $T$ is the characteristic function of $K_{p} M K_{p}=\coprod M_{i} K_{p}$, and if $\Phi$ is right $K_{p}$-invariant, then

$$
(T \Phi)(g)=\sum \Phi\left(g M_{i}\right)
$$

In the proof of the following lemma we use the notation $M^{*}=\mu(M) M^{-1}$, which is an anti-involution of $G$. Note that

$$
\Gamma M \Gamma=\Gamma M^{*} \Gamma,
$$

since $M$ may be chosen to be diagonal ( $M \mapsto M^{*}$ is not the identity on diagonal matrices, but operates as conjugation with $J$, which is an element of $\Gamma$ ).

Lemma 9. The lifting $f \longmapsto \Phi_{f}$ defined by (23) is Hecke-equivariant, i.e.,

$$
T \Phi_{f}=\Phi_{f \mid T} \quad \text { for each } T \in \mathcal{H}\left(G_{p}, K_{p}\right)
$$

(here we identified the $p$-adic and the classical Hecke algebra according to Lemma 8).

Proof. It is enough to check that both sides are equal when evaluated at an element $g \in G_{\infty}^{+}$. We may further assume that $T$ is a double coset,

$$
T=\Gamma M \Gamma=\coprod_{i} \Gamma M_{i} \quad \text { with } M_{i} \in G\left(\mathbb{Z}\left[p^{-1}\right]\right)^{+} .
$$

By the above considerations, we have $K_{p} M K_{p}=\coprod K_{p} M_{i}$, and therefore

$$
K_{p} M K_{p}=K_{p} M^{*} K_{p}=\coprod_{i} M_{i}^{*} K_{p}
$$

For a matrix $M \in G(\mathbb{Q})$ we write $M_{\infty}$ for the matrix considered as an element of $G_{\infty}$, and $M_{p}$ considered as an element of $G_{p}$. With these notations, we have

$$
\begin{aligned}
\left(T \Phi_{f}\right)(g) & =\sum_{i} \Phi_{f}\left(g\left(M_{i}^{*}\right)_{p}\right)=\sum_{i} \Phi_{f}\left(\left(M_{i}^{*}\right)_{\mathbb{Q}}^{-1} g\left(M_{i}^{*}\right)_{p}\right) \\
& =\sum_{i} \Phi_{f}\left(\left(M_{i}^{*}\right)_{\infty}^{-1} g\right)=\sum_{i}\left(f \mid\left(M_{i}^{*}\right)^{-1} g\right)(I) \\
& =\sum_{i}\left(f \mid M_{i} g\right)(I)=((f \mid T) \mid g)(I)=\Phi_{f \mid T}(g) . \square
\end{aligned}
$$

It follows from this lemma and (30) that

$$
T \Phi_{f}=\operatorname{det}(M)^{(n+1-k) / 2} \Phi_{f \| T} \quad \text { for } T=\Gamma M \Gamma .
$$

The following theorem summarizes the results of the previous sections. 
Theorem 1. The mapping $f \mapsto \Phi_{f}$ defined by (23) maps the space $S_{k}\left(\Gamma_{n}\right)$ of classical Siegel cuspforms of degree $n$ and weight $k$ isometrically (see Lemma 6) and in a Hecke-equivariant way ( see Lemma 9) into a subspace of $L_{0}^{2}(Z(\mathbb{A}) G(\mathbb{Q}) \backslash G(\mathbb{A})$ ) consisting of continuous functions $\Phi$ on $G(\mathbb{A})$ with the following properties:

i) $\Phi(\varrho g)=\Phi(g) \quad$ for $\varrho \in G(\mathbb{Q})$,

ii) $\Phi\left(g k_{0}\right)=\Phi(g)$ for $k_{0} \in K_{0}=\prod_{p<\infty} G\left(\mathbb{Z}_{p}\right)$,

iii) $\Phi\left(g k_{\infty}\right)=\Phi(g) j\left(k_{\infty}, I\right)^{-k}$ for $k_{\infty} \in K_{\infty} \simeq U(n)$,

iv) $\Phi(g z)=\Phi(g)$ for $z \in Z(\mathbb{A})$, the center of $G(\mathbb{A})$,

v) $\Phi$ is smooth as a function of $G(\mathbb{R})^{+}$(fixed finite components), and is annihilated by $\mathfrak{p}_{\mathbb{C}}^{-}$(see Lemma 7$)$,

vi) $\Phi$ is cuspidal (see Lemma 5).

\subsection{The associated representation}

We now associate an automorphic representation of $\operatorname{PGSp}(2 n)$ with a Hecke eigenform of degree $n$.

Let $f$ be a cuspidal Hecke eigenform of degree $n$ and weight $k$. Let $\Phi_{f}$ be the associated function on $G(\mathbb{A})$, defined by (23). Then $\Phi_{f}$ is an automorphic form on $G(\mathbb{A})$ which lies in $L^{2}(Z(\mathbb{A}) G(\mathbb{Q}) \backslash G(\mathbb{A}))$, and in fact in the cuspidal subspace $L_{0}^{2}(Z(\mathbb{A}) G(\mathbb{Q}) \backslash G(\mathbb{A}))$ by Lemma 5. Denote by $V_{f}$ the subspace of this $L_{0}^{2}$-space spanned by all right translates of $\Phi_{f}$. Let $\pi$ be any irreducible constituent of this unitary representation (it is well known that $L_{0}^{2}(Z(\mathbb{A}) G(\mathbb{Q}) \backslash G(\mathbb{A})$ ) decomposes discretely into irreducible components). Then $\pi$ is an automorphic representation of $G(\mathbb{A})$ which is trivial on $Z(\mathbb{A})$. We may thus consider $\pi$ as an automorphic representation of $\operatorname{PGSp}(2 n, \mathbb{A})$.

Let

$$
\pi=\bigotimes_{p} \pi_{p}
$$

be the decomposition of $\pi$ into irreducible representations $\pi_{p}$ of the local groups $G_{p}=G\left(\mathbb{Q}_{p}\right)$ (restricted tensor product). Because $\Phi_{f}$ is right $K_{p}=G\left(\mathbb{Z}_{p}\right)$ invariant at every finite place $p$, the representation $\pi_{p}$ is spherical for every such $p$. As discussed in 3.2, it is of the form $\pi\left(\chi_{0}, \ldots, \chi_{n}\right)$ for certain unramified characters $\chi_{i}$ of $\mathbb{Q}_{p}^{*}$, with Satake parameters $\beta_{i}:=\chi_{i}(p)$.

We also have the classical Satake parameters of the eigenform $f$, defined as follows (see $[\mathrm{An}])$. There is a character $\lambda \in \operatorname{Hom}_{\mathrm{Alg}}\left(\mathcal{H}_{p}^{\text {class }}, \mathbb{C}\right)$, such that

$$
f \|_{k} T=\lambda(T) f
$$

It is known that there are non-zero complex numbers $a_{0}, \ldots, a_{n}$ such that

$$
\lambda(\Gamma M \Gamma)=a_{0}^{\delta} \sum_{i} \prod_{j=1}^{n}\left(a_{j} p^{-j}\right)^{d_{i j}},
$$


where $\Gamma M \Gamma=\bigsqcup_{i} \Gamma M_{i}$ and

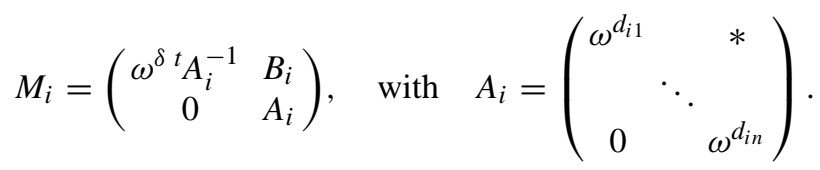

The $a_{0}, \ldots, a_{n}$ are the classical Satake p-parameters of the eigenform $f$.

Lemma 10. If $a_{0}, a_{1}, \ldots, a_{n}$ are the classical Satake p-parameters of $f$, then

$$
p^{n(n+1) / 4-n k / 2} a_{0}, a_{1}, \ldots, a_{n}
$$

are the Satake parameters of the spherical representation $\pi_{p}$, the local component of $\pi$ at $p$.

Proof. With $T=\Gamma M \Gamma$, by (31) we have

$$
\begin{aligned}
T \Phi_{f} & =\operatorname{det}(M)^{(n+1-k) / 2} \lambda(T) \Phi_{f}=\mu(M)^{n(n+1-k) / 2} \lambda(T) \Phi_{f} \\
& =p^{\delta n(n+1-k) / 2} a_{0}^{\delta} \sum_{i} \prod_{j=1}^{n}\left(a_{j} p^{-j}\right)^{d_{i j}} \Phi_{f} .
\end{aligned}
$$

Thus $T \Phi_{f}=\tilde{\lambda}(T) \Phi_{f}$ with

$$
\tilde{\lambda}(T)=p^{\delta n(n+1) / 4} b_{0}^{\delta} \sum_{i} \prod_{j=1}^{n}\left(b_{j} p^{-j}\right)^{d_{i j}},
$$

where $b_{0}=p^{n(n+1) / 4-n k / 2} a_{0}$ and $b_{j}=a_{j}$ for $j=1, \ldots, n$. It follows from (10) that $b_{0}, \ldots, b_{n}$ are the Satake parameters of $\pi_{p}$ (note that if $M_{i}$ is of the form (33), then $M_{i}^{*}$ is of the form (7)).

It follows from (11) that for the classical Satake parameters we have

$$
a_{0}^{2} a_{1} \cdot \ldots \cdot a_{n}=p^{k n-n(n+1) / 2}
$$

a relation also found in $[\mathrm{AK}]$.

We shall now describe the local component $\pi_{\infty}$ in the tensor product decomposition (32) of $\pi=\pi_{f}$. This component will only depend on the weight $k$ of the modular form $f$, which is a positive integer. Recall the definition (19) of the torus elements $T_{i} \in \mathfrak{k}_{\mathbb{C}}$.

Lemma 11. The representation $\pi_{\infty}$ of $G(\mathbb{R})$ contains a smooth vector $v_{\infty}$ with the following properties:

i) $\pi\left(k_{\infty}\right) v_{\infty}=j\left(k_{\infty}, I\right)^{-k} v_{\infty}$ for all $k_{\infty} \in K_{\infty}$.

ii) $T_{i} v_{\infty}=k v_{\infty}$ for all $i=1, \ldots, n$.

iii) $\mathfrak{p}_{\mathbb{C}}^{-} v_{\infty}=0$. 
Proof. In $\pi$ the vector $\Phi_{f} \in V_{f}$ projects to a pure tensor $v=\otimes_{p \leq \infty} v_{p}$ with $v_{p} \in$ $\pi_{p}$ a spherical vector for finite $p$, and $v_{\infty} \in \pi_{\infty}$ inheriting the analytic properties of $\Phi_{f}$. Thus i) follows from Theorem 1 iii). Then ii) follows by a straightforward computation. Finally iii) follows from Theorem $1 \mathrm{v}$ ).

By this lemma, $\pi_{\infty}$ is an irreducible unitary representation of $\operatorname{PGSp}(2 n, \mathbb{R})$ with a lowest weight vector of weight $k\left(e_{1}+\ldots+e_{n}\right)$. There is only one such representation, namely the representation $\pi_{k}$ constructed in 3.5 . To summarize,

Theorem 2. Let $\pi$ be the automorphic representation of $\operatorname{PGSp}(2 n, \mathbb{A})$ associated with $f \in S_{k}\left(\Gamma_{n}\right)$, as described at the beginning of this section. Then the local components $\pi_{p}$ of $\pi$ are given as follows:

i) At the archimedean place, $\pi_{\infty}$ is the lowest weight representation $\pi_{k}$ constructed in 3.5 (it is a holomorphic discrete series representation exactly for $k>n$, and a limit of discrete series for $k=n$ ).

ii) At a finite place, $\pi_{p}$ is the spherical principal series representation (see Sect. 3.2) of $\operatorname{PGSp}\left(2 n, \mathbb{Q}_{p}\right)$ with Satake parameters $p^{n(n+1) / 4-n k / 2} a_{0}, a_{1}, \ldots, a_{n}$, where $a_{1}, \ldots, a_{n}$ are the classical Satake parameters of the modular form $f$.

\subsection{Vector valued modular forms}

For completeness we shall indicate in this section how to extend the lifting procedure described in the previous sections to vector valued Siegel modular forms. A good source for vector valued modular forms is [Fr2]. The lifting procedure for these forms is briefly described in [We].

Let $(\rho, W)$ be a finite-dimensional rational representation of $\operatorname{GL}(n, \mathbb{C})$. Rational means that there is an integer $k$ such that the representation $A \mapsto \operatorname{det}(A)^{-k} \rho(A)$ is polynomial (holomorphic). A vector valued modular form of type $\rho$ and degree $n$ is a holomorphic function $f: \mathbb{H}_{n} \rightarrow W$ with the property that

$$
f(\gamma\langle Z\rangle)=\rho(C Z+D) f(Z) \quad \text { for all } \gamma=\left(\begin{array}{ll}
A & B \\
C & D
\end{array}\right) \in \Gamma_{n}, Z \in \mathbb{H}_{n} .
$$

For the one-dimensional representation $\rho(A)=\operatorname{det}(A)^{k}$ we recover the usual scalar valued modular forms. Cuspidality is defined by properties of Fourier coefficients, as in the scalar case. The space of all cusp forms of type $\rho$ and degree $n$ shall be denoted by $S_{\rho}\left(\Gamma_{n}\right)$.

Assuming $\rho$ is irreducible, we now associate a function $\Phi$ on the adele group to a modular form $f$, as follows. Let $m \in \mathbb{R}$ be the number such that $\rho(s)=s^{2 m} \operatorname{id}_{W}$, for each scalar matrix $s=\operatorname{diag}(s, \ldots, s) \in \operatorname{GL}(n, \mathbb{C}), s>0$ (if $\rho=\operatorname{det}^{k}$, then $m=n k / 2)$. Then we define

$$
\tilde{\Phi}(g)=\mu\left(g_{\infty}\right)^{m} \rho(C I+D)^{-1} f(g\langle I\rangle),
$$

with $g=g_{\mathbb{Q}} g_{\infty} k_{0} \in G(\mathbb{A})$ as in $(22)$ and $g_{\infty}=\left(\begin{array}{ll}A & B \\ C & D\end{array}\right)$. 
This function is well-defined in view of (34). The factor $\mu\left(g_{\infty}\right)^{m}$ ensures that $\tilde{\Phi}$ descends to a function on $\operatorname{PGSp}(2 n, \mathbb{A})$. We further have $\tilde{\Phi}\left(\gamma g k_{0}\right)=\tilde{\Phi}(g)$ for all $\gamma \in G(\mathbb{Q})$ and $k_{0} \in K_{0}$, and

$$
\tilde{\Phi}\left(g k_{\infty}\right)=\rho\left(k_{\infty}\right)^{-1} \tilde{\Phi}(g) \text { for all } k_{\infty} \in K_{\infty} \simeq U(n),
$$

where the identification $K_{\infty} \simeq U(n)$ is given by $\left(\begin{array}{cc}A & B \\ -B & A\end{array}\right) \mapsto A-i B$. Just as in Lemma 7 one proves that the holomorphy of $f$ is equivalent to $\mathfrak{p}_{\mathbb{C}}^{-} \cdot \tilde{\Phi}=0$.

On the group one would like to have scalar valued functions lying in the usual $L^{2}$-space, instead of the vector valued $\tilde{\Phi}$. Therefore let $L$ be any non-zero linear form on $W$, the space of $\rho$, and define

$$
\Phi(g)=L(\tilde{\Phi}(g)), \quad g \in G(\mathbb{A}) .
$$

We will eventually consider the space generated by all right translates of $\Phi$, and therefore the choice of $L$ is irrelevant.

The Petersson scalar product on $S_{\rho}\left(\Gamma_{n}\right)$ is given by

$$
\left\langle f_{1}, f_{2}\right\rangle=\int_{\Gamma_{n} \backslash \mathbb{H}_{n}}\left\langle\rho\left(Y^{1 / 2}\right) f_{1}(Z), \rho\left(Y^{1 / 2}\right) f_{2}(Z)\right\rangle d^{*} Z .
$$

Here the inner product $\langle$,$\rangle is a U(n)$-invariant hermitian form on $W$ (unique up to scalars), and $Y^{1 / 2}$ denotes the unique positive definite square root of the positive definite matrix $Y$. Note that this is a generalization of the Petersson scalar product in (25).

$$
\text { For } g=\left(\begin{array}{ll}
A & B \\
C & D
\end{array}\right) \in \operatorname{Sp}(2 n, \mathbb{R}) \text { and } Z=g\langle I\rangle \text { we have } Y=\left(C{ }^{t} C+D^{t} D\right)^{-1}=
$$
$\left(M^{t} \bar{M}\right)^{-1}$, where $M=C I+D$. Write $M=p u$ with a symmetric positive $p$ and a unitary $u$. Then $p^{-1}=Y^{1 / 2}$, and there exists a constant $c>0$ such that

$$
\begin{aligned}
\left\|\rho\left(Y^{1 / 2}\right) f(Z)\right\|^{2} & =\left\|\rho(M)^{-1} f(Z)\right\|^{2}=\|\tilde{\Phi}(g)\|^{2} \\
& =c \int_{K_{\infty}}\left|L\left(\rho(k)^{-1} \tilde{\Phi}(g)\right)\right|^{2} d k=c \int_{K_{\infty}}|\Phi(g k)|^{2} d k .
\end{aligned}
$$

Now integration yields

$$
\begin{aligned}
\int_{\Gamma_{n} \backslash \mathbb{H}_{n}}\left\|\rho\left(Y^{1 / 2}\right) f(Z)\right\|^{2} d^{*} Z & =c \int_{Z(\mathbb{A}) G(\mathbb{Q}) \backslash G(\mathbb{A}) / K} \int_{K_{\infty}}|\Phi(g k)|^{2} d k d g \\
= & c \int_{Z(\mathbb{A}) G(\mathbb{Q}) \backslash G(\mathbb{A})}|\Phi(g)|^{2} d k d g .
\end{aligned}
$$

This shows that, after suitable normalization of measures, the map $f \mapsto \Phi$ is a norm-preserving map of Hilbert spaces from $S_{\rho}\left(\Gamma_{n}\right)$ to

$$
L^{2}(Z(\mathbb{A}) G(\mathbb{Q}) \backslash G(\mathbb{A})) .
$$


The image is contained in the space of cuspidal functions, cf. Lemma 5.

Assuming that $f$ is a Hecke eigenform, one can now associate an automorphic representation $\pi_{f}$ of $\operatorname{PGSp}(2 n, \mathbb{A})$ with $f$ as in the previous section. The space of $\pi_{f}$ will be an irreducible subspace of the $G(\mathbb{A})$-space generated by $\Phi$. For each place $p$ one can characterize the local components $\pi_{p}$ of $\pi_{f}$ as in Theorem 2 . We shall indicate what is different now at the archimedean place $p=\infty$.

It follows from (36) that the $U(n)$-module $\tau$ generated by $U(n)$-translates of $\Phi$ is isomorphic to the contragredient of $\left.\rho\right|_{U(n)}$ (note that $\left.\rho\right|_{U(n)}$ remains irreducible by the unitary trick). Furthermore, $\Phi$ has the lowest weight property because $f$ is holomorphic. Consequently, the local component $\pi_{\infty}$ is the lowest weight representation of $G(\mathbb{R})$ with minimal $K_{\infty}$-type $\tau$ (more precisely, since $G(\mathbb{R})$ is disconnected, $\pi_{\infty}$ combines a lowest and a highest weight representation).

The irreducible representations of $K_{\infty} \simeq U(n)$ are parameterized by elements in the weight lattice $\Lambda=\mathbb{Z} e_{1}+\ldots+\mathbb{Z} e_{n}$, modulo the action of the real Weyl group $W_{r}$. Since $W_{r} \simeq S_{n}$ acts by permuting the coefficients of the $e_{i}$, the irreducible representations of $K_{\infty}$ are in 1-1 correspondence with the weights

$$
\lambda=r_{1} e_{1}+\ldots+r_{n} e_{n}, \quad r_{i} \in \mathbb{Z}, r_{1} \geq \ldots \geq r_{n} .
$$

We denote the finite-dimensional irreducible representation corresponding to this $\lambda$ by $\tau_{\lambda}$ or $\tau_{r_{1}, \ldots, r_{n}}$. The correspondence is that $\tau_{\lambda}$ contains a vector $v_{0}$ that is annihilated by the compact positive root vectors, and such that $T_{j} v_{0}=r_{j} v_{0}$ (see (19)). Thus $v_{0}$ is a highest weight vector.

The irreducible rational representations of $\operatorname{GL}(n, \mathbb{C})$ are also parameterized by integers $r_{1} \geq \ldots \geq r_{n}$. If $\rho_{r_{1}, \ldots, r_{n}}$ denotes the corresponding representation, parameterized as in [Fr2] appendix to I.6, then one checks that $\left.\rho_{r_{1}, \ldots, r_{n}}\right|_{U(n)}=$ $\tau_{-r_{n}, \ldots,-r_{1}}$, assuming the identification $K_{\infty} \simeq U(n)$ fixed above. In other words, the contragredient of $\left.\rho_{r_{1}, \ldots, r_{n}}\right|_{U(n)}$ is $\tau_{r_{1}, \ldots, r_{n}}$.

It follows that if $f$ is a cusp form of type $\rho_{r_{1}, \ldots, r_{n}}$ in the sense of [Fr2], then $\pi_{\infty}$ is the representation of $G(\mathbb{R})$ with minimal $K_{\infty}$-type $\tau_{r_{1}, \ldots, r_{n}}$. In the scalar valued case we have $r_{1}=\ldots=r_{n}=k$. If $r_{n}>n$, then $\pi_{\infty}$ is a holomorphic discrete series representation with Harish-Chandra parameter $\left(r_{1}-1\right) e_{1}+\ldots+\left(r_{n}-n\right) e_{n}$, cf. Sect. 3.5.

\subsection{L-functions}

Let $f$ be a cuspidal Siegel eigenform, and let $\pi_{f}$ be the automorphic representation of $\operatorname{PGSp}(2 n, \mathbb{A})$ associated with $f$ as in the previous section. The dual group of $\operatorname{PGSp}(2 n)$ is $\operatorname{Spin}(2 n+1)$. In Sect. 3.4 we considered two finite-dimensional representations of $\operatorname{Spin}(2 n+1, \mathbb{C})$, the "projection representation" $\varrho_{1}$, and the $2^{n}$-dimensional spin representation $\varrho_{2}$.

Corresponding to these two representations of the connected component of the $L$-group, we have two Langlands $L$-functions associated with $\pi_{f}$. According to our results in Sect. 3.4, they are given by

$$
L\left(s, \pi_{f}, \varrho_{1}\right)=\prod_{p}\left(\left(1-p^{-s}\right) \prod_{i=1}^{n}\left(1-b_{i} p^{-s}\right)\left(1-b_{i}^{-1} p^{-s}\right)\right)^{-1},
$$


and

$$
L\left(s, \pi_{f}, \varrho_{2}\right)=\prod_{p}\left(\prod_{k=0}^{n} \prod_{1 \leq i_{1}<\ldots<i_{k} \leq n}\left(1-b_{0} b_{i_{1}} \ldots b_{i_{k}} p^{-s}\right)\right)^{-1},
$$

where $b_{0}, b_{1}, \ldots, b_{n}$ are the Satake parameters of the local component $\pi_{f, p}$. But by Lemma 10, these Satake parameters are essentially the same as the classical Satake parameters of the eigenform $f$. In view of the definitions (1) and (2) of the classical $L$-functions, we obtain the following result.

Theorem 3. Let $\pi_{f}$ be the cuspidal automorphic representation of the group $\operatorname{PGSp}(2 n, \mathbb{A})$ associated with the cuspidal Siegel eigenform $f$ of degree $n$ and weight $k$. Then for the standard L-function of $f$ we have the identity

$$
L_{1}(s, f)=L\left(s, \pi_{f}, \varrho_{1}\right),
$$

where $\varrho_{1}$ is the projection representation $(14)$ of $\operatorname{Spin}(2 n+1, \mathbb{C})$, and for the spin $L$-function of $f$ we have

$$
L_{2}\left(s^{\prime}, f\right)=L\left(s, \pi_{f}, \varrho_{2}\right), \quad s^{\prime}=s-n(n-1) / 4+n k / 2,
$$

where $\varrho_{2}$ is the $2^{n}$-dimensional spin representation of $\operatorname{Spin}(2 n+1, \mathbb{C})$.

We also remark that in the case $n=2$ there is an isomorphism $\operatorname{Spin}(5, \mathbb{C}) \simeq$ $\operatorname{Sp}(4, \mathbb{C})$, and the spin representation becomes the standard representation of $\operatorname{Sp}(4, \mathbb{C})$.

This result allows us to carry over general group theoretic results to classical $L$-functions. As an example, we prove the meromorphic continuation of the spin $L$-functions for cuspforms in $S_{k}\left(\Gamma_{3}\right)$ using Langlands's theory of Euler products.

Let $\mathbf{M}$ be a maximal standard Levi subgroup in a connected reductive Chevalley group G. Consider $\mathbf{G}$ as a group over $\mathbb{Q}$. (However, this theory is available in more generality, cf. [Sh], for example.) Let $\mathbf{P}=\mathbf{M N}$ be a standard parabolic in $\mathbf{G}$. Denote by $\hat{P}=\hat{M} \hat{N}$ the parabolic in $\hat{G}$, the complex dual of $\mathbf{G}$, corresponding to $\mathbf{P}$ (cf. [Bo]). Let $r$ denote the adjoint action of $\hat{M}$ on the Lie algebra of $\hat{N}$ and write $r=\oplus_{i=1}^{m} r_{i}$, with $r_{i}$ 's the irreducible constituents of $r$.

Let $\pi=\otimes_{p} \pi_{p}$ be a cuspform on $M=\mathbf{M}(\mathbb{A})$. Let

$$
f=\otimes_{p} f_{p} \in I(s, \pi)=\otimes_{p} I\left(s, \pi_{p}\right),
$$

with the same notation as in Sect. 2 of [Sh]. Let $S$ be a finite set of places for which every $\pi_{p}$ with $\pi \notin S$ is unramified. The theory of Euler products developed by Langlands (cf. [La]) then implies that

$$
M(s, \pi) f=\left(\prod_{p \in S} A\left(s, \pi_{p}\right) f_{p}\right) \otimes\left(\otimes_{p \notin S} \tilde{f}_{p}\right) \cdot \prod_{i=1}^{m} \frac{L_{S}\left(i s, \pi, \tilde{r}_{i}\right)}{L_{S}\left(1+i s, \pi, \tilde{r}_{i}\right)}
$$

has a meromorphic continuation to all of $\mathbb{C}$. For our purposes we may assume $S=$ $\{\infty\}$. The intertwining operator $A\left(s, \pi_{\infty}\right)$ is non-vanishing and has meromorphic continuation to all of $\mathbb{C}$ (cf. Section 17 of [KS]). This is true even if $S$ contains finite places (cf. [Sh]). 
Now assume $m=1$. From the above discussion and (37), it follows that

$$
F(s)=\frac{L\left(s, \pi, \tilde{r_{i}}\right)}{L\left(s+1, \pi, \tilde{r_{i}}\right)}
$$

is meromorphic. Writing this as

$$
L\left(s, \pi, \tilde{r_{i}}\right)=F(s) L\left(s+1, \pi, \tilde{r_{i}}\right)
$$

and noting that $L\left(s, \pi, \tilde{r}_{i}\right)$ is analytic if $\operatorname{Re}(s)$ is sufficiently large, one concludes by induction that $L\left(s, \pi, \tilde{r_{i}}\right)$ has a meromorphic continuation to all of $\mathbb{C}$. In particular, we get the following.

Theorem 4. The L-function $L\left(s, \pi_{f}, \varrho_{1}\right)$ of Theorem 3 has a meromorphic continuation to all of $\mathbb{C}$.

Proof. Consider $\mathbf{M}=\mathrm{GL}(1) \times \mathrm{Sp}(6)$ as a standard Levi subgroup in $\mathbf{G}=\mathrm{Sp}(8)$. The dual of $\mathbf{M}$ is $\mathbb{C}^{\times} \times \operatorname{SO}(7, \mathbb{C})$ with $m=1$ and $r=r_{1}$ is seven dimensional (cf. Case $\left(C_{n}\right)$ of Sect. 4 of [Sh]). Now, if we put the representation $\sigma=\mathbf{1} \otimes \pi_{f}$ on $M=\mathbf{M}(\mathbb{A})$, then, since $r_{1}$ is self-contragredient, $L\left(s, \pi_{f}, \varrho_{1}\right)=L\left(s, \sigma, r_{1}\right)$, which has meromorphic continuation to all of $\mathbb{C}$ by our previous argument.

To prove a similar result for the other $L$-function consider the following. Let $\mathbf{G}$ be a Chevalley group of type $F_{4}$. This is a split (as well as adjoint) simply connected simple algebraic group with Dynkin diagram

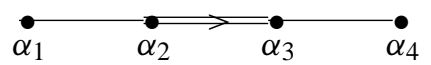

Consider the standard parabolic subgroup $\mathbf{P}=\mathbf{M N}$, where $\mathbf{M}$ is the standard Levi subgroup corresponding to $\left\{\alpha_{2}, \alpha_{3}, \alpha_{4}\right\}$. One can then show that $\mathbf{M} \simeq \operatorname{GSp}(6)$ (cf. [As]). Note that the complex dual of $\mathbf{G}$ is again of type $F_{4}$ and contains the dual parabolic $\hat{P}=\hat{M} \hat{N}$ (see [Bo]). In fact,

$$
\hat{M}=\operatorname{GSpin}(7, \mathbb{C})
$$

is the dual of M. This is the case (xxii) of Sect. 6 of [La] with $m=2$ and we have $r=r_{1} \oplus r_{2}$ with $r_{1}$ an eight dimensional and $r_{2}$ a seven dimensional representation of $\hat{M}$. Indeed, if $r$ is restricted to $\operatorname{Spin}(7, \mathbb{C}) \subset \hat{M}$, then $r_{1}$ is the eight dimensional spin representation while $r_{2}$ is what we called the projection representation in Sect. 3.4. Note that these representations are self-dual, and therefore we do not have to care about contragredients in the following.

Put a cuspform $\pi$ on $M=\mathbf{M}(\mathbb{A})$. Now (37) and its subsequent argument again imply that

$$
G(s)=\frac{L\left(s, \pi, r_{1}\right)}{L\left(s+1, \pi, r_{1}\right)} \cdot \frac{L\left(2 s, \pi, r_{2}\right)}{L\left(2 s+1, \pi, r_{2}\right)}
$$

has a meromorphic continuation to all of $\mathbb{C}$. Write this as

$$
L\left(s, \pi, r_{1}\right)=G(s) \cdot \frac{L\left(2 s+1, \pi, r_{2}\right)}{L\left(2 s, \pi, r_{2}\right)} \cdot L\left(s+1, \pi, r_{1}\right) .
$$


Now if we let $\pi$ be the representation on $M=\operatorname{GSp}(6, \mathbb{A})$ lifted up from $\pi_{f}$ on $\operatorname{PGSp}(6, \mathbb{A})$, then Theorem 4 and an induction similar to its proof imply the following.

Theorem 5. The L-function $L\left(s, \pi_{f}, \varrho_{2}\right)$ of Theorem 3 has a meromorphic continuation to all of $\mathbb{C}$ if $n=3$.

Hence, in view of Theorem 3, we get

Corollary 1. Let $f \in S_{k}\left(\Gamma_{3}\right)$ be a cuspform of degree 3 , and let $L_{2}(s, f)$ be the spin L-function attached to $f$. Then $L_{2}(s, f)$ has meromorphic continuation to all of $\mathbb{C}$.

We remark that the same proof works for vector valued modular forms.

Acknowledgements. We would like to thank Professor F. Shahidi for his help with this work. The first author, in particular, is grateful to him for suggesting and supervising his thesis problem which inspired some of the ideas in the current work. The final stage of this work by the first author was conducted for the Clay Mathematics Institute. The second author was supported by Deutscher Akademischer Austauschdienst DAAD.

\section{References}

[An] Andrianov, A.: Euler products associated with Siegel modular forms of degree two. Russ. Math. Surveys 29, 3, 45-116 (1974)

[AK] Andrianov, A., Kalinin, V.: On the analytic properties of standard zeta functions of Siegel modular forms. Math. USSR-Sb. 35, 1-17 (1979)

[AZ] Andrianov, A., Zhuravlev, V.: Modular Forms and Hecke Operators. AMS Translations of Mathematical Monographs 145, 1995

[As] Asgari, M.: On the holomorphy of local Langlands $L$-functions. Ph.D. thesis, Purdue University, 2000

[Bö] Böcherer, S.: Über die Funktionalgleichung automorpher $L$-Funktionen zur Siegelschen Modulgruppe. J. reine angew. Math. 362, 146-168 (1985)

[Bo] Borel, A.: Automorphic $L$-functions. Proc. Symp. Pure Math. 33, 2, $27-61$ (1979)

[Bu] Bump, D.: Automorphic Forms and Representations. Cambridge: Cambridge University Press, 1997

[Ca] Cartier, P.: Representations of p-adic groups: A survey. Proc. Symp. Pure Math. 33, 1, 111-155 (1979)

[Fr1] Freitag, E.: Siegelsche Modulfunktionen. Heidelberg: Springer, 1983

[Fr2] Freitag, E.: Singular modular forms and theta relations. Lecture Notes in Mathematics 1487, Berlin-Heidelberg-New York: Springer, 1991

[FH] Fulton, W., Harris, J.: Representation Theory. Berlin-Heidelberg-New York: Springer, Graduate Texts in Mathematics 129

[Ge] Gelbart, S.: Automorphic Forms on Adele Groups. Ann. Math. Studies 83, Princeton, 1975

[GS] Gelbart, S., Shahidi, F.: Analytic Properties of Automorphic $L$-functions. LondonNew York: Academic Press, 1988

[K1] Klingen, H.: Introductory Lectures on Siegel Modular Forms. Cambridge: Cambridge University Press, 1990 
[Kna] Knapp, A.: Representation Theory of Semisimple Groups. An Overview Based on Examples. Princeton University Press, 1986

[KS] Knapp, A., Stein, E.: Intertwining operators for semisimple groups. Ann. Math. 93, 2, 489-578 (1971)

[Kne] Kneser, M.: Strong approximation. Proc. Symp. Pure Math. 9, 187-196 (1966)

[La] Langlands, R.: Euler products. Yale University Press, 1971

[Sh] Shahidi, F.: On the Ramanujan conjecture and finiteness of poles for certain Lfunctions. Ann. Math. 127, 547-584 (1988)

[Sp] Springer, T.: Reductive groups. Proc. Symp. Pure Math. 33, 1, 3-27 (1979)

[We] Weissauer, R.: Vektorwertige Siegelsche Modulformen kleinen Gewichtes. J. reine angew. Math. 343, 184-202 (1983) 